\title{
Using Eye Tracking to Understand the Impact of Cognitive Abilities on Search Tasks
}

\author{
Efi A. Nisiforou, Eleni Michailidou, and Andrew Laghos \\ Department of Multimedia and Graphic Arts \\ Cyprus University of Technology, Limassol, Cyprus \\ \{efi.nisiforou, eleni.michailidou, andrew.laghos\}@cut.ac.cy
}

\begin{abstract}
Nowadays, there is an increase of studies that examine individuals' cognitive characteristics in correlation to visual perception. The present study investigated the association between cognitive abilities and Web page complexity. Specifically, differences within simple, medium and complex Web pages were observed among the field dependent, independent and mixed cognitive groups via a task completion time with the use of the eye tracking technology. The results showed that task completion time is significantly different in medium and complex pages between the FD and FI users, while, in the simple pages, no statistical differences appeared. Furthermore, it was supported that users' FD-I cognitive construct style can be identified using innovative techniques like eye tracking studies by analyzing users' scan path and heat maps.
\end{abstract}

Keywords: Field Dependent-Independent, cognitive abilities, visual complexity, ViCRAM algorithm, eye tracking.

\section{Introduction}

The visual appearance of interactive systems, such as Web pages and e-learning environments, tend to convey more information than one can imagine. Contact points that users make first to perceive such a system affect the rest of the interaction process [1]. Individuals' cognitive characteristics and Web pages visual complexity have been gaining ground in the literature. This study is a pilot investigation towards a project that aims to formulate a framework and suggest guidelines in designing adaptive environments by understanding how users of different cognitive types interact with different tasks [2]. The use of eye tracking technology as a measure of noticing users' cognitive ability during visual processing will be investigated as a methodology in achieving the above objective. Cognitive style data is being incorporated into adaptive systems for the development of personalized user models. The link between eye tracking and cognitive modelling is an extremely intuitive and fruitful area of research. It is important therefore, to understand precisely what the eyes reveal in order to model human behavior by designing suitable and adaptive environments based on the assumption that individuals interact differently. Developing a new objective method of 
measuring the cognitive behavior of humans, instead of solely relying on questionnaires, is of paramount importance. Finally, by understanding sighted users' visual understanding of Web page complexity in relation with the time of task completion, important information should reveal with refer to the cognitive effort required for interaction with that page.

A previous pilot study examined the potential of eye tracker as a tool in detecting users' cognitive dimensions with respect to the FD-I classification and identified differences between the three cognitive construct styles and tasks time completion [2]. It was mentioned that complexity consists an interesting concept with its applications to be used in for a wide range of uses, from cognitive psychology to computer science [3]. An earlier study conducted by Michailidou [4] found that visually complex pages generate users' disoriented navigation while, visually simple pages produce the opposite perspective with the use of the ViCRAM algorithm. This presented study was designed to investigate whether eye tracking can assess subjects' differences on cognitive dimensions and examine the correlation between these styles and tasks time completion. Specifically, it attempts to identify users' cognitive ability based on their Web behavior and relate individuals' cognitive characteristics such as visual attention patterns in terms of field dependent-independent construct style with Web pages' visual complexity. The use of eye tracking technology as a measure of noticing users' cognitive ability during visual processing gives insight to how this information and cognitive overload affects user ability to interact.

Therefore, the following research question is addressed:

1. How does users' Web behavior in terms of task time completion differ in simple, medium and complex Web pages among FD, FI and FN users?

\section{Related Work}

Access to and movement around complex hypermedia environments has long been considered an important issue in the Web design and usability field [5]. With the rapid and constant advancement of technology, new ways are continually being introduced to present information that leads to visually complex Web pages. Cognitive overload is a result of the boost of information presented on the Web. The information on most of these Web pages is visually fragmented and organized into groups [6]. In this way, when users reach a Web page, they can scan the page and obtain a comprehension of it in a few seconds. Cognition refers to the ability of the human mind to acquire and manage information [7] and comprises different mental processes such as attention, memory, perception, problem solving and learning [8]. Moreover, it describes the tendencies as the modes in which humans approach, acquire, organize, process, interpret information [9] and how they use these interpretations to guide their actions [10]. Visual perception encompasses complex cognitive processes that are involved in other forms of conceptualization and learning [11]. 


\subsection{Field Dependent-Independent}

The field dependent-independent (FDI) construct lies within the most broadly studied of a variety of cognitive style dimensions appearing in the literature and especially in the educational technology field [12]. These dimensions are formed based on the individual's reliance on the context to extract particular meaning and describe users. The key difference between FD and FI learners is visual perceptiveness. FD learners, who are asked to identify a simple geometric figure that is embedded in a complex figure, will take longer time to detect the simple figure than FI learners, or they may not be able to find it at all. On the contrary, FI learners have difficulty in abstracting relevant information from visual instructional materials. In line with the results of previous work $[12,13]$, it is hypothesized that FI learners will outperform FD learners as well in terms of problem-solving performance.

Much of the research on FD-I dimension has focused on examining the effects of FD-I on learners' computer performance [14]. A study by Ford, Miller, and Moss [15] demonstrated individual cognitive-style differences in Web searching tasks. It is important to note that most studies rely heavily on the prior completion of questionnaires by system users. Since the completion of questionnaires can be time consuming for users, potentially improving the measurement methods of users' cognitive load is meaningful.

\subsection{Visual Complexity}

Complexity can be defined as "the degree of difficulty in providing a verbal description of an image" [16]. Textures with repetitive and uniform oriented patterns are less complex than disorganized ones. A visual pattern is also described as complex if its parts are difficult to identify and separate from each other [16]. Complexity perception of an image depends on the amount of grouping, the quantity of the parts an observer perceives in the scene, familiarity with the scene and existing knowledge of objects inside the scene. Visual complexity is mainly represented by the perceptual dimensions of quantity of objects, clutter, openness, symmetry, organization, and the variety of colors $[16,17]$.

Studies try to identify Web page design metrics that predict whether a site is highly rated with regard to complexity $[18,19]$. These studies relate Web site design guidelines with complexity explaining that the way a Web site is presented depends on the way the page itself is designed and what elements (metrics) are used; Web page code complexity was not examined. Others [19] propose cognition, content and form as the three primary factors that affect the complexity of a Web site. Tuch et al. [20] mentioned that visual complexity of Websites has several effects on human cognition and emotion.

The above studies [18-20] tried to develop techniques to empirically examine all aspects of Web site design, but without being able to define and investigate visual complexity. ViCRAM [4], which is the algorithm employed in this study, used the afore-mentioned characteristics and findings concentrated on Web page design and the structure that defines visually simple and complex interfaces. The specific 
algorithm was used since it was based both on users' perception and the underlying structure of the page.

\subsection{Eye Tracking}

Cognitive and semantic aspects of a stimulus play an important role in visual and scene perception [17]. Eye movements are driven by properties of the visual world and processes in a person's mind [17]. Eye tracking and usability evaluation studies try to investigate and understand user behavior $[17,21]$ with an increasing interest to Web page behavior. A general conclusion is that user interaction depends on the visual factors (nearby visual features) and scene semantics (general knowledge about the scene layout). The idea that user' features such as cognitive abilities and personality are affecting the effectiveness of information visualization techniques is continuously growing. An eye tracking study conducted by Toker et al. [22] investigated the relationship between such characteristics and fine-grained user attention patterns. Their findings revealed that user's cognitive abilities such as verbal working memory and perceptual speed have a significant impact on gaze behavior in terms of visualization type and task difficulty.

\section{$3 \quad$ Methodology}

A comparative evaluation of two methodologies was conducted: users' cognitive abilities identification based on the Field Dependent-Independent classification using Hidden Figures Test (HFT) and Web page visual complexity using an automatic algorithm (ViCRAM). It was hypothesized that field independent learners' will need less time in identifying a task in a Webpage, whereas, field dependent learners' will take more time in completing a task and therefore a more disoriented behavior will be occurred. In addition, participants' visual attention was measured and analyzed based on the scan path and heat maps eye gaze analysis. Then results from both studies were compared to answer this pilot study's hypothesis.

\subsection{Participants}

The population used in the study was taken from a Public University in Cyprus (age range 18 - 28). The participants were initially categorized into 7 FD, 6 FI and 3 FN/FM learners using the Hidden Figures Test (HFT).

\subsection{Procedure}

The study was conducted in two parts:

Part A - Hidden Figure Test - Participants level of field independent was measured with the use of the Hidden Figures Test developed by Witkin et al. [23]. Participants' had a 30 minutes time limit to complete the test. 
Part B - User Evaluation: Cognitive Abilities - Users' interaction behavior was examined using eye tracking as a tool to assess their cognitive abilities. Participants were placed in front of an eye tracker and asked to complete a task on 9 Web pages that were preselected. Nine fact-finding tasks or known- item search task (tasks in which the information is located in a particular place of a Webpage) were defined in the experiment in searching for a specific information. Participants' had to click on the finding task as to indicate their response. All 9 Web pages were analysed using the ViCRAM algorithm, and visual complexity score was given for each page. Due to the number of pages used, complexity was described in three categories: Simple, Medium and Complex.

\subsection{Materials}

The researchers' administered the HFT from the Educational Testing Services kit for cognitive factors designed by Witkin et al. [23] to measure participants' cognitive ability and classify them into a field type. It consists of 32 questions divided equally into two parts. The test presents five simple figures and asks learners to find one of the 5 simple figures embedded in a more complex pattern. Those possessing a score 10 or below they were defined as FD, while FI scored 16 or higher and FN scored from 11 to 15 .

The stimuli used during the eye tracking study contained 9 Web pages with a range of visual complexity from 0 - 10 (0 visual simple and 10 visual complex) based on the ViCRAM tool. For the data analysis, Complexity categorization was used which was retrieved based on the algorithm rankings (see Table 1). The content of the Web pages retrieved from five different categories: Shopping, Government, Leisure-Social, Education and News.

Table 1. Web Pages Used: Category and Complexity Level

\begin{tabular}{lll}
\hline Page ID & Category & $\begin{array}{l}\text { Complexity } \\
\text { Level }\end{array}$ \\
\hline P1 & Government & Simple \\
\hline P2 & Leisure/Social & Simple \\
\hline P3 & Education & Simple \\
\hline P4 & Leisure/Social & Medium \\
\hline P5 & Education & Medium \\
\hline P6 & Education & Medium \\
\hline P7 & News & Complex \\
\hline P8 & News & Complex \\
\hline P9 & Government & Complex \\
\hline
\end{tabular}




\section{$4 \quad$ Results and Discussion}

Eye movements were recorded during task processing with the aid of the eye tracker iViewX model software. The Web stimulus recording mode of the BeGaze 3.1 analysis software was used to capture not only the eye movements, but also mouse clicks as a way of detecting users' task time completion. Participants' visual attention was measured and analyzed based on the scan path and heat maps eye gaze analysis. Finally, users' cognitive dimensions as retrieved from the HFT and task completion time and were statistically analyzed with the use of the SPSS. The findings of the study are discussed with respect to how FD, FM, and FI learners behave within simple, medium and complex pages.

\subsection{Hidden Figure Test}

As previously mentioned, the Hidden Figure Test (HFT) was used to determine users' main current cognitive occupation (e.g. field dependent, field independent and field neutral/mixed) in terms of their level of field independence. Their score on the test was calculated as the difference between the number of questions answered correctly minus the number answered incorrectly. Taking into how other researchers' determined the cut-off scores of the test [24, 25], participants were classified as 7 Field Dependence, 6 Field Independence, and 3 as Field Mixed learners. The testing activity involved in the HFT has been described as perceptual disembedding and is a reliable and widely used approach for determining FD-I [26]. Kuder-Richardson reliability coefficient of the Hidden Figure Test reflects the degree of .76 [27].

\subsection{Task Completion Time}

Participants' had to click on the finding task by indicating their response and thus identifying the amount of completion time of each participant per task. This variable was taken into account since researchers were aiming to examine how subjects' cognitive trait with respect to time task completion affects visual complexity. Since the number of participants was small and this being a pilot study, a comparison between the average completion time for each complexity categorization and cognitive ability was calculated. Therefore, for the statistical test, for each complexity level (simple, medium, complex) the average completion time was calculated, ending up with three completion times. Then Levene's test equality of variances was performed to examine the relation between cognitive abilities and time completion.

The results of this test (see Table 1) indicated that completion time is significantly different in complex pages between the FD and FI users, [ $\mathrm{t}(12)=2.34, \mathrm{p}=.04$ ], as the first group produced significantly higher time completion means $(M=157.33$, $\mathrm{SD}=72.60)$ than the FI group $(\mathrm{M}=84.70, \mathrm{SD}=38.53)$. The same pattern was observed in the medium pages, showing a significant difference in terms of time task completion between the two groups, $[\mathrm{t}(6.82)=3.82, \mathrm{p}=.007]$ with the FD group 
$(\mathrm{M}=101.84, \mathrm{SD}=34.46)$ outperforming the users' of the FI group $(\mathrm{M}=50.45$, $\mathrm{SD}=9.01)$. Additionally, in the simple pages no statistical differences appeared. A possible assumption might rely on the fact that people behave the same or perhaps any differences in the behavior are less likely to have an effect on a simple complexity page, since, the layout of the pages does not impede users' navigation and Web search. Comparisons between the FN group and the other two groups were not statistically significant at $\mathrm{p}<0.05$.

Table 2. Independent sample T-test with regard to users' cognitive ability scores and webpages' visual complexity

\begin{tabular}{|c|c|c|c|c|c|c|}
\hline \multirow{2}{*}{\multicolumn{2}{|c|}{ Levene's Test Equality of Variances }} & \multicolumn{5}{|c|}{ t-test for Equality of } \\
\hline & & $\mathrm{F}$ & Sig. & $\mathrm{t}$ & df & $\begin{array}{c}\text { Sig. } \\
\text { (2-tailed) }\end{array}$ \\
\hline \multirow{3}{*}{ a) Complex } & & & & & & \\
\hline & Equal val rames assumea & 3.821 & 0.014 & 2.538 & 12 & $0.038^{*}$ \\
\hline & Equal variances not assumed & & & 2.338 & 9.132 & 0.044 \\
\hline \multirow{2}{*}{ b) Medium } & Equal variances assumed & 7.028 & 0.021 & 3.817 & 12 & 0.002 \\
\hline & Equal variances not assumed & & & 3.817 & 6.816 & $0.007 *$ \\
\hline \multirow[b]{2}{*}{ c) Simple } & Equal variances assumed & 3.601 & 0.082 & 1.407 & 12 & 0.185 \\
\hline & Equal variances not assumed & & & 1.407 & 7.461 & 0.200 \\
\hline
\end{tabular}

*The mean difference is significant at the 0.05 level.

In line with these results, a study by Nisiforou and Laghos [2] found a large variation in task completion time among the FD-I cognitive groups. Similar findings were observed in the work conducted by Burnett et al [28], as the FI learners' outperformed FD learners in terms of time taken to respond correctly to a problem-solving task. Other studies stated that FI individuals face less difficulty in separating the most essential information from its context than FD subjects do [29].

\subsection{Cognitive Abilities and Visual Complexity}

Users' cognitive behavior based on their field dependent-independent dimension was analyzed through gaze plots (scan path) and attention maps (focus map, heat map).

The results revealed that although participants were engaged in the same online environments of viewing activity, they tend to demonstrate different attention patterns. The stimulated data indicated that users' cognitive abilities with respect to visual attention show a significant impact on gaze behaviour. The emerged results were discussed in view of common and different Web navigation behaviour with regard to the three cognitive ability categories. According to Figure 1 users' common behavior within a simple page is revealed without taking into account their level of field independent. This observation supports the previous results, since no statistical differences exist in the simple pages among the three cognitive groups. 


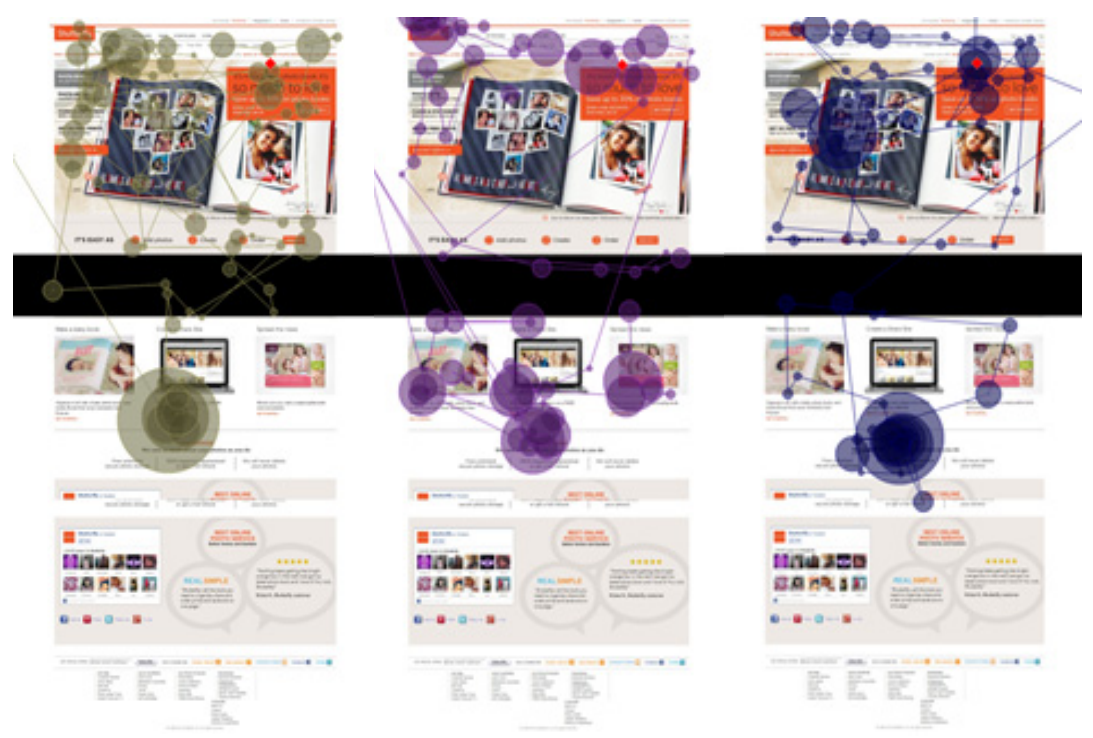

Fig. 1. Gaze Plot/Scanpaths demonstrating the common behaviour on simple pages FI, FD and FN (from left to right)

On the contrary, Figures 2 and 3 demonstrated evidence on how users' cognitive characteristics affect their Web navigation according to medium and complex pages respectively. Besides, FD users' scan paths appeared to be more disoriented and scattered on visual complex pages in contrast to FI subjects' that displayed a more oriented and organized scan paths. Similar results were reported in a study conducted by Harper et al. [6]. In line with this, Michailidou [4] found that visually complex pages generate users' disoriented navigation while, visually simple pages produce the opposite perspective.

Since the perception of complexity is correlated with the variety in the visual stimulus a visual pattern may also look complex if its parts are difficult to identify and separate from each other [3]. Data analysis revealed that users' cognitive ability has a significant impact on user gaze behavior and that this influence is detectable through a variety of eye tracking metrics.

The results also highlight the importance of designing environments that reflect individuals' cognitive characteristics. The environments could be e-learning or any Web pages and interfaces in general for which a user can adapt based on their characteristics. These outcomes are in line with Toker et al. [22] that investigated the relationship of users' such characteristics along with their attention patterns. Individuals that are located towards the FD dimension have difficulty in separating incoming information from its contextual surroundings, and are more likely to be influenced by external cues and to be non-selective in their information uptake. FI individuals on the other hand, are more likely to be influenced by internal than external cues and therefore, be selective in their information input [28, 29]. 
Many of the research on FD-I has focused on examining the effects of FD-I on learners' computer performance [14]. A study conducted by Jozsa [30] demonstrated that the cognitive style is related to differences in the Web searching tasks. In an earlier study, it was recommended that further studies are needed with respect to subjects' cognitive style [24]. It is important to note that since the completion of surveys is time consuming; potentially improving the measurement methods of users' cognitive load is also significant.

Additionally, the results suggest that the cognitive ability classification may be predicted by the time completion of each task. In line with these results, Tinajero et al. [31] found that field independent students generally performed better than fielddependent students.

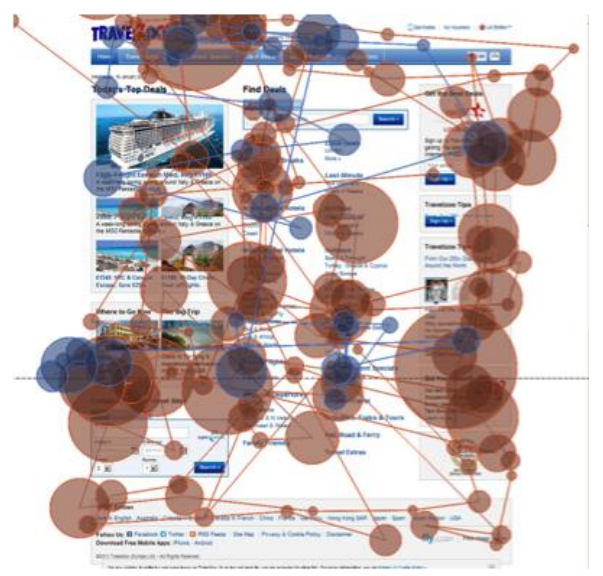

Fig. 2. Scanpaths showing the non-common behaviour of FD (orange) and FI (blue) subjects in a medium page

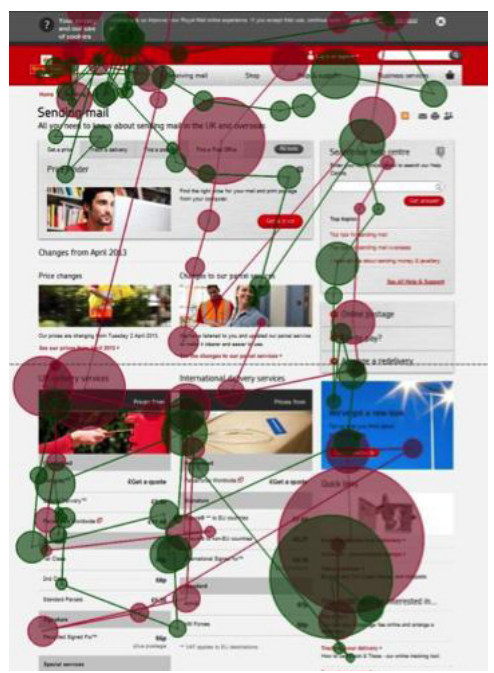

Fig. 3. Scanpaths indicating the different behaviour between FD (green) and FI (red) users in complex page

The complexity algorithm was explicitly used in this study as a tool that allowed the investigation between eye tracking technologies and cognitive abilities. Specifically, with both these technologies (algorithm and eye tracking) this pilot study identified that: 1) Field dependency could be determined with the use of eye tracking technology, 2) Visual complexity prediction tools could be used to evaluate an interface in order to help in the interface adaptation, 3) Designers will have an additional tool for validating their interface in order to design environments that not only assist users with disabilities, but all users that would like to interact with interfaces that meet their learning and interaction style. 


\section{Conclusions}

This study is a pilot investigation towards a project that aims to formulate a framework and suggest guidelines in designing adaptive environments by understanding how users of different cognitive types interact with different tasks [2]. It supports the view that individual differences exist in terms of cognitive abilities that should provide insight to users' navigation in assessing and perusing digital information. This demonstrates that the differences among the behavior of the FD and FI cognitive groups, in terms of the time taken to complete the given tasks in the complex and medium complex Webpages should be taken into consideration.

Cognitive style ultimately has implications for the design of hypertext instructional systems and the development of personalized user models. The use of eye tracker technology as a measure of noticing users' cognitive ability during visual processing gives an understanding on how this information and cognitive overload affects user ability to interact. The link between eye tracking and cognitive modeling is extremely intuitive and fruitful area of research. It is important, therefore, to understand precisely what the eyes reveal in order to model human behavior by designing suitable and adaptive environments based on the assumption that individuals interact differently. Developing a new measurement method to test the cognitive behavior of humans, instead of solely relying on questionnaires, is of paramount importance. This can lead to solutions that improve users' Web experience. Finally, the results of the study demonstrate a necessity for emerging solutions that will reflect the user's cognitive ability and can, however, improve users' Web experience through the design of innovative interfaces.

In addition, further research is under progress that will take the above study on its next stage using neuroimaging methodologies such as Electroencephalography (EEG). EEG is a tool that allows us to detect the location and the changes in the brain activity while it is performing a cognitive task. Hence, the design of guidelines will be conducted to be used by developers in order to create simpler Web pages to allow interaction for all cognitive abilities. The findings should provide insights that enable the development of models that better predict and stimulate human performance in evaluating cognitive load.

\section{References}

1. Lindgaard, G., Fernandes, G., Dudek, C., Browntilde, J.: Attention web designers: You have 50 milliseconds to make a good first impression! Behaviour \& Information Technology $25,115-126$ (2006)

2. Nisiforou, E.A., Laghos, A.: Do the eyes have it? Using eye tracking to assess students' cognitive dimensions. Educational Media International 50, 247-265 (2013)

3. DaSilva, M., Courboulay, V., Estraillier, P.: Image complexity measure based on visual attention. In: IEEE International Conference on Image Processsing, ICIP 2011, pp. 32813284 (2011)

4. Michailidou, E.: ViCRAM Visual complexity Rankings and accessibility Metrics. PhD thesis, The University of Manchester (2009) 
5. Furuta, R., Frank, M., Shipman, I., Marshall, C.C., Brenner, D., wei Hsieh, H.: Hypertext paths and the world-wide web: experiences with walden's paths. In: HYPERTEXT 1997, pp. 167-176. ACM, New York (1997)

6. Harper, S., Michailidou, E., Stevens, R.: Toward a definition of visual complexity as an implicit measure of cognitive load. ACM Trans. Appl. Percept. 6, 1-18 (2009)

7. Germanakos, P., Tsianos, N., Lekkas, Z., Mourlas, C., Samaras, G.: Eye-tracking users' behavior in relation to cognitive style within an e-learning environment. In: ICALT 2009, pp. 329-333 (2009)

8. Solso, R.L., MacLin, M.K., MacLin, O.H.: Cognitive Psychology, 7th edn. Allyn \& Bacon (2004)

9. Messick, S.: The nature of cognitive styles: Problems and promise in educational practice. Educational Psychologist 19, 59-74 (1984)

10. Hayes, J., Allinson, C.W.: Cognitive style and the theory and practice of individual and collective learning in organizations. Human Relations 51, 847-871 (1998)

11. Workman, M.: Performance and perceived effectiveness in computer-based and computeraided education: do cognitive styles make a difference? Computers in Human Behavior 20, 517-534 (2004)

12. Dragon, K.: Field dependence and student achievement in technology-based learning: A meta-analysis. Master's thesis, University of Alberta (2009)

13. Burnett, W.C.: Cognitive style: A meta-analysis of the instructional implications for various integrated computer enhanced learning environments. $\mathrm{PhD}$ thesis, Indiana University of Pennsylvania (2010)

14. Hercegfi, K.: Event related assessment of hypermedia based e-learning materials with an HRV-based method that considers individual differences in users. International Journal of Occupational Safety and Ergonomics 17, 119-127 (2011)

15. Ford, N., Miller, D., Moss, N.: Web search strategies and human individual differences: Cognitive and demographic factors, Internet attitudes and approaches. Journal of the American Society for Information Science and Technology 56, 741-756 (2005)

16. Oliva, A., Mack, M.L., Shrestha, M., Peeper, A.: Identifying the perceptual dimensions of visual complexity in scenes. In: 26th Annual Meeting of the Cognitive Science Society, Chicago (2004)

17. Rayner, K.: Eye movements in reading and information processing: 20 years of research. Psychological Bulletin 124, 372-422 (1998)

18. Ivory, M.Y., Sinha, R.R., Hearst, M.A.: Empirically validated web page design metrics. In: CHI 2001, pp. 53-60. ACM Press, New York (2001)

19. Germonprez, M., Zigurs, I.: Causal factors for web site complexity. Working Papers on Information Environments, Systems and Organizations 3 (2003)

20. Tuch, A.N., Bargas-Avila, J.A., Opwis, K., Wilhelm, F.H.: Visual complexity of websites: Effects on users' experience, physiology, performance, and memory. Int. J. Hum.-Comput. Stud. 67, 703-715 (2009)

21. Jacob, R.J.K.: Eye tracking in advanced interface design. Oxford University Press Inc., NY (1995)

22. Toker, D., Conati, C., Steichen, B., Carenini, G.: Individual user characteristics and information visualization: connecting the dots through eye tracking. In: CHI 2013, pp. 295304. ACM Press, NY (2013)

23. Witkin, H.A., Moore, C.A., Goodenough, D.R., Cox, P.W.: Field-dependent and fieldindependent cognitive styles and their educational implications. Review of Educational Research 47, 1-64 (1977) 
24. French, J.W., Ekstrom, R.B., Price, L.A.: Kit of reference tests for cognitive skills. Educational Testing Services, Princeton (1963)

25. Angeli, C.: Examining the effects of field dependence-independence on learners' problemsolving performance and interaction with a computer modeling tool: Implications for the design of joint cognitive systems. Computers \& Education 62, 221-230 (2013)

26. Rittschof, K.A.: Field dependence-independence as visuospatial and executive functioning in working memory: implications for instructional systems design and research. Educational Technology Research and Development 58, 99-114 (2010)

27. Study, N.E.: An overview of tests of Cognitive spatial ability. Paper presented at 66th Midyear Meeting Proceedings. ASEE, Galveston (2012)

28. Guisande, M., Pramo, M., Tinajero, C.Y., Almeida, L.: Field dependence-independence (fdi) cognitive style: An analysis of attentional functioning. Psicothema 19, 572-577 (2007)

29. Zhang, L.: Field-dependence/independence: Cognitive style or perceptual ability? validating against thinking styles and academic achievement. Personality and Individual Differences 37, 1295-1311 (2004)

30. Jozsa, E., Hamornik, B.P.: Find The Difference! Eye Tracking Study on Information Seeking Behavior Using an Online Game. Journal of Eye Tracking, Visual Cognition and Emotion 2, 27-35 (2012)

31. Tinajero, C., Pramo, M.F.: Field dependence-independence and academic achievement: a re-examination of their relationship. British Journal of Educational Psychology 67, 199212 (1997) 